\title{
The engineered Salmonella typhimurium inhibits tumorigenesis in advanced glioma
}

This article was published in the following Dove Press journal:

OncoTargets and Therapy

15 September 2015

Number of times this article has been viewed

\author{
Jian-qiang Chen' \\ Yue-fu Zhan ${ }^{2}$ \\ Wei Wang' \\ Sheng-nan Jiang ${ }^{2,3}$ \\ Xiang-ying $\mathrm{Li}^{2}$
}

'Department of Radiology, The Third Xiangya Hospital, Central South University, Changsha, People's Republic of China; ${ }^{2}$ Department of Radiology, Affiliated Haikou Hospital of Xiangya School of Medicine, Central South University, Haikou, Hainan, People's Republic of China; ${ }^{3}$ Department of Nuclear Medicine, Affiliated Haikou Hospital of Xiangya School of Medicine, Central South University, Haikou, Hainan, People's Republic of China
Correspondence: Wei Wang Department of Radiology, The Third Xiangya Hospital, Central South University, I72 Tongzipo Road, Changsha, Hunan 4100I3,

People's Republic of China Email cjr.wangwei@vip.l63.com

Sheng-nan Jiang

Department of Nuclear Medicine, Affiliated Haikou Hospital of Xiangya School of Medicine, Central South University, 43 Renmin Road, Haikou, Hainan 570208, People's Republic of China

Email quanjz@hanmail.net
Objective: To explore the antitumor role of the attenuated Salmonella typhimurium $\Delta \mathrm{ppGpp}$ with inducible cytolysin $A(C l y A)$ in advanced stage of glioma.

Materials and methods: The C6 rat glioma cells were orthotopically implanted by surgery into the caudate nucleus of rat brains. The rats were then randomly divided into the treatment group (SL + ClyA) ( $n=12)$, negative control group (SL) ( $n=12)$, and control group (phosphatebuffered saline $[\mathrm{PBS}])(\mathrm{n}=12)$. In the treatment group, the attenuated $S$. typhimurium were transformed with the plasmid-encoded antitumor gene ClyA. The expression of ClyA was controlled by the TetR-regulated promoter in response to extracellular doxycycline. The plasmid also contained an imaging gene $l u x$ to allow illumination of the tumor infected by the bacteria. The rat glioma C6 cells were implanted into the caudate nucleus of all rats. The engineered S. typhimurium and respective controls were injected intravenously into the rats 21 days after initial tumor implantation. The pathological analysis of the glioma tumor was performed at 21 days and 28 days ( 7 days after doxycycline treatment) postimplantation. All rats underwent MRI (magnetic resonance imaging) and bioluminescence study at 21 days and 28 days postimplantation to detect tumor volume. The differences between the three groups in tumor volume and survival time were analyzed.

Results: Advanced stage glioma was detected at 21 days postimplantation. Bioluminescence showed that the engineered S. typhimurium accumulated in glioma tumors and disappeared in the normal reticuloendothelial tissues 3 days after intravenous injection. MRI showed that the tumor volume in the S. typhimurium with ClyA group were significantly reduced compared to the bacteria alone and no bacteria groups 7 days post-doxycycline treatment $(P<0.05)$, while the necrotic tumor volume in the $S$. typhimurium with ClyA group and $S$. typhimurium alone group increased significantly compared to the control group $(P<0.01)$. In addition, the survival time was significantly prolonged in the bacteria-treated group compared to the PBS-treated control group $(P<0.01)$.

Conclusion: The engineered $S$. typhimurium can significantly induce cancer cell apoptosis in the tumor center and inhibit cancer cell proliferation in the outer zone of advanced glioma tumor, leading to a prolonged survival time in rats. In addition, the engineered S. typhimurium that carried the antitumor and imaging genes controlled by the TetR-regulated promoter have high delivery efficiency with tolerable side effects in rats.

Keywords: Salmonella typhimurium, cytolysin A, brain tumor, advanced glioma

\section{Introduction}

Malignant gliomas are the most common primary central nervous system (CNS) tumors and account for $78 \%$ of overall CNS tumors in adults. ${ }^{1}$ The current standard treatment for malignant glioma often includes a combination of surgical removal, radiotherapy, and chemotherapy. ${ }^{2}$ However, the prognosis of glioma remains very poor, especially in patients with advanced stage of gliomas. ${ }^{3}$ The median survival time for advanced gliomas is merely $8-12$ months and the 5 -year survival rate is only $2.5 \%-5 \% .{ }^{4}$ One of 
the most challenging problems in the treatment of advanced gliomas is the highly invasive nature of gliomas in their advanced stage. Currently, the development of novel antiglioma therapeutics has been focused on preventing the invasion of advanced glioma. ${ }^{4}$

Bacterial treatment of cancer has unique advantages compared to other anticancer treatments. The engineered bacteria can specifically target the tumors and induce controllable cytotoxicity in cancer cells..$^{5-7}$ Nowadays, the biosynthetic technology has allowed us to solve many problems that limited the use of bacterial therapy in the past, such as the toxicity, stability, and efficiency, making the bacterial therapy a promising anticancer therapy. ${ }^{7}$ In particular, the engineered bacteria has the following advantages in the treatment of advanced glioma: first, the blood-brain barrier (BBB) is severely damaged in the advanced glioma, which makes intravenous (IV) injection of bacteria possible to reach therapeutic concentration in the CNS since most bacteria penetrate the BBB poorly. ${ }^{8-10}$ In addition, IV injection is more practical and acceptable because it does not require the complex craniotomy operation and thus reduces the risk of causing bacterial infections when compared to the intracranial administration of bacteria. ${ }^{9,10}$ Second, advanced gliomas are highly proliferative and invasive. ${ }^{10}$ Bacterial therapy designs bacteria that has the capability to work as a robot inside the cancer cells because they have intact biological machinery to carry out the antitumor functions. $^{7}$

Traditionally, the bacterial strain used in the treatment of cancer targets the center of a tumor and spares the outer proliferation zone of a tumor. In this study, we engineered a bacteria strain that contains an anticancer gene that can inhibit cancer cell proliferation in the tumor proliferation zone. In this way, the engineered bacteria can have simultaneous effects on inducing apoptosis in the tumor center and inhibiting proliferation and invasion in the tumor outer layer. To use bacteria as a carrier to deliver the antitumor gene requires a regulatory mechanism that can precisely control gene expression at the right time and right location. We thus used a repressor-regulated doxycycline efflux system to control the expression of the antitumor gene in bacteria, which is turned on in response to extracellular doxycycline. ${ }^{11,12}$ The attenuated Salmonella typhimurium was integrated with an imaging reporter gene $(\operatorname{lux})^{13,14}$ and transformed by a plasmid-encoded antitumor gene $(C l y A)^{12,15}$ to lyse tumor cells once expressed inside the cancer cells. We used the bioluminescent technology to track the accumulated sites of the engineered bacteria in rats, which allow us to examine the antitumor effects of the bacteria by the tet on/off system to control the antitumor gene expression in an advanced glioma rat model.

\section{Materials and methods Cell culture}

The rat C6 glioma cells were obtained from the cell bank of the Chinese Academy of Sciences (Shanghai, People's Republic of China). Cells were cultured at $37^{\circ} \mathrm{C}$ and $5 \%$ carbon dioxide in Dulbecco's Modified Eagle's Medium $\left(\right.$ Gibco $^{\circledR}$, Life Technologies, Carlsbad, CA, USA) supplemented with $10 \%$ fetal bovine serum and $100 \mathrm{U} / \mathrm{mL}$ penicillin (Hyclone Laboratories, Inc., Logan, UT, USA). Cells were cultured till $80 \%-90 \%$ confluence before being harvest.

\section{Animal model}

All animal experiments were approved by the Central South University Animal Research Committee. Thirty-six healthy adult male Sprague Dawley rats weighing 220-250 g were anesthetized and placed in a stereotaxic frame (RWD Life Science, Shenzhen, People's Republic of China). A burr hole was drilled in the skull $1 \mathrm{~mm}$ anterior to the bregma and $3 \mathrm{~mm}$ lateral to the midline. ${ }^{15}$ The bone fragments from a $5 \mathrm{~mm}$ diameter hole were removed and subcutaneous cleared. The microsyringe needles advanced to the depth of $5 \mathrm{~mm}$ and $1 \times 10^{6} \mathrm{C} 6$ glioma cells suspended in $10 \mu \mathrm{L}$ of phosphatebuffered saline (PBS) were injected. ${ }^{16}$

\section{Preparation of engineered S. typhimurium}

The primers, plasmids, and bacterial strains (S. typhimurium $\Delta$ ppGpp/lux) used in this study were kindly provided by the Chonnam National University, South Korea. S. typhimurium $\Delta$ ppGpp/lux-pTet/ClyA (S.t- $\left.\Delta \mathrm{pG}^{\text {lux/pT-ClyA }}\right)$ were constructed as described previously. ${ }^{11,12} \mathrm{We}$ constructed the plasmid pJL43 based on the plasmid pJL39. The antitumor gene $(C l y A)$ was placed under the control of the TetR system in the pJL43 plasmid and was then transformed into the $S$. typhimurium $\Delta \mathrm{ppGpp/lux} \mathrm{strains.} \mathrm{All} \mathrm{the} \mathrm{primers} \mathrm{and} \mathrm{constructs}$ used in this study are listed in Tables 1 and 2.

The S. typhimurium- $\Delta \mathrm{pG}^{\mathrm{lux} / \mathrm{pT}-\mathrm{ClyA}}$ was streaked on the LuriaBertani plate which contained ampicillin and kanamycin. A single colony was picked out and grown overnight in lysogeny broth medium on a shaking incubator $\left(37^{\circ} \mathrm{C}, 220 \mathrm{rpm}\right)$. Then it was diluted 50-fold into fresh medium and grown to early stationary phase, and harvested by centrifugation (5,000 rpm, 10 minutes), quantified by spectrophotometry, and diluted with PBS to desired concentration for step experiments. ${ }^{17}$ 
Table I Primers used in this study

\begin{tabular}{lll}
\hline Target & Primer & Template \\
\hline tetRA & RltetR, 5'-TTAAGACCCACTTTCACATT-3' & TH9952 \\
& RltetA, 5'-CTAAGCACTTGTCTCCTG-3' & PJL30A \\
RBS-MCS I & TetPXbalF, 5'-ACTTTTATCTAATCTAGACATCA-3' & \\
& TetPXbalrev, 5'-GCCGCCATGGCCCGGGATCCTGCAGGCCTTCT & PJL30A \\
RBS-MCS II & CTATCACTGATAGGGAGT-3' & \\
& TetRNrulF, 5'-TACTAAGTCATCGCGATGGAGCAA-3' & \\
ClyA(A) & TerRNrulrev, 5'-AATCCTCGACAGGCTTCTCGAGTGGCCACTCC & \\
& TGCTTAAGACCCACTTTCACATTTAAGT-3' & PAClyA \\
\hline
\end{tabular}

\section{Experimental design}

The advanced stage glioma formed at 21 days postimplantation, ${ }^{18}$ and the animals were then randomly divided into the treatment group, the negative control group, and the control group. The rats in the treatment group were injected with $100 \mu \mathrm{L}$ PBS containing $1 \times 10^{8} \mathrm{CFU} / \mathrm{mL}$ of $S . t-\Delta \mathrm{pG} \mathrm{l}^{\mathrm{lux} / \mathrm{pT}-\mathrm{ClyA}}$ via the tail vein, and named the SL + ClyA group $(n=12)$. The rats in the negative control group were injected with $100 \mu \mathrm{L}$ PBS containing $1 \times 10^{8} \mathrm{CFU} / \mathrm{mL} S$. typhimurium, and named as the SL group $(n=12)$. The control group was named as the PBS group $(n=12)$ and were injected with $100 \mu \mathrm{L}$ PBS buffer only.

\section{MRI and optical bioluminescence imaging}

Magnetic resonance imaging (MRI) was performed with a 3.0 T MRI system (Signa HDxt; GE Healthcare Bio-Sciences Corp., Piscataway, NJ, USA) using a small animal head coil (Shanghai Chenguang Medical Technologies Co. Ltd, Shanghai, People's Republic of China). The MRI sequences and parameters were: T2-weighted sequence (echo train length $=4$; repetition time $=3,000 \mathrm{~ms}$; echo time $=120 \mathrm{~ms}$; number of averages $=1$ ) and T1-weighted sequence (repetition time $=350 \mathrm{~ms}$; echo time $=$ minimum value; number of average $=2$ ) before and after injection of $0.4 \mathrm{~mL}$ contrast medium (gadodiamide, Omniscan; Amersham Health,
Princeton, NJ, USA). Images were acquired for eight slices in the axial plane ( slice thickness $=2.0 \mathrm{~mm}$; matrix $=192 \times 192$ ). Rats were anesthetized by isoflurane (initial dose $2.5 \%$, maintenance $1.6 \%$ ). To assess the tumor and necrosis volume, MRI was performed in rats at 21 and 28 days post-tumor implantation using the MRIcro software. Bioluminescence imaging was performed with the IVIS 100 system (Caliper Life, Sciences, Hopkinton, MA, USA). ${ }^{12,15,18}$ ImageJ was used to measure tumor volume and necrotic volume.

\section{Doxycycline administration and safety evaluation}

Doxycycline hyclate (Doxy; Sigma-Aldrich Co., St Louis, MO, USA) was administrated orally at a total amount of $17 \mathrm{mg} / \mathrm{kg}$ according to previous study. ${ }^{12,19}$ The timing of doxycycline administration was determined when the S.t- $\Delta \mathrm{pG}$ lux/pT-ClyA have accumulated in the tumor and cleared from the normal tissues. The body weight of rats were measured every day until 35 days postimplantation in order to evaluate the safety profile of $S . t-\Delta \mathrm{pG}$ lux/pT-ClyA to normal tissues.

\section{Western blot analysis}

After resecting C6 tumor from the rats, tumors were homogenized in RIPA buffer (Sigma-Aldrich Co.) containing protease

Table 2 Plasmids and Salmonella typhimurium strains used in this study

\begin{tabular}{|c|c|}
\hline Strains/plasmids & Description \\
\hline pGEM-T vector & Purchased from Promega \\
\hline PJL30A & PGEM-T vector encode into the "tetRA" of PCR product \\
\hline PJL32 & Deleted TetA gene of pJL30A and encode "RBS-MCS I" of PCR product under the TetA promoter \\
\hline PJL37 & Encode the 'RBS-MCS II' of PCR product into PGEM-T vector \\
\hline PJL39 & $\begin{array}{l}\text { pJL32 and pJL37 were digested by Nru I and Sca I restriction enzymes, the construction of pJL39 was ligated } \\
\text { by the small fragment of pJL32 and large fragment of pJL37 }\end{array}$ \\
\hline pJL43 (pT-ClyA) & PJL39 encode into cytolysin A under TetA promoter \\
\hline S.t- $\Delta p G^{\operatorname{lux}}$ & S. typhimurium $\Delta \mathrm{pp} G \mathrm{pp} / \mathrm{lux}$ \\
\hline S.t- $\Delta p G^{\operatorname{lux} / p T-C l y A}$ & PJL43 was transformed into S.t- $\Delta$ pG lux \\
\hline
\end{tabular}

Abbreviation: PCR, polymerase chain reaction. 
inhibitor cocktail (Sigma-Aldrich Co.) on ice. After 120 minutes incubation and 30 minutes centrifugation $\left(12,000 \mathrm{rpm}\right.$ at $\left.4{ }^{\circ} \mathrm{C}\right)$, the supernatant was then pipetted off and kept on ice. Protein concentrations were measured by bicinchoninic acid assay kit (Bio-Rad Laboratories Inc., Hercules, CA, USA).

Protein samples were subjected to sodium dodecyl sulfate-polyacrylamide gel electrophoresis on $12 \%$ linear gradient gels and transferred to nitrocellulose membranes (Bio-Rad) at $110 \mathrm{~V}$ for 3 hours. The membranes were washed with Tris-buffered saline ( $\mathrm{pH} 7.4$ ) containing $0.1 \%$ Tween 20 (TBS-T) and blocked for 2 hours with 5\% nonfat milk in TBS-T. The membranes were then incubated for 2 hours at room temperature with ClyA antibody (at 1:250 dilution, gift from Piao Hong-Hua, Central South University, Changsha, People's Republic of China) and the others with an actin antibody (at 1:2,000 dilution, Sigma-Aldrich Co.) in TBS-T with $2 \%$ nonfat milk. They were again incubated for 2 hours. Protein bands were detected with ClyA/actin antibodies using HRP-conjugated, anti-rabbit secondary antibody (Amersham, UK) in TBS-T with $2 \%$ nonfat milk. The protein levels were determined using enhanced chemiluminescence plus (Amersham, Buckinghamshire, UK) and Image Reader (LAS-3000 Imaging System; Fuji Photo Film, Tokyo, Japan).

\section{Histopathological analysis}

Histopathological study conducted at 21 and 28 days posttumor implantation from rats randomly selected in each of the three groups. In brief, the tumor was resected and fixed with 4\% paraformaldehyde overnight. Representative sections ( $4 \mu \mathrm{m}$ thickness) were stained with hematoxylin-eosin (H\&E). For immunofluorescent staining, the slides were incubated with anti-ClyA (1:80) and antisalmonella (1:100, Abcam, Cambridge, UK) overnight. On the next day, donkey anti-rabbit 555 (1:100, Thermo Fisher Scientific, Waltham,
MA, USA) or donkey anti-rat 488 (1:100, Thermo Fisher Scientific) was used as the secondary antibody and incubated for 2 hours. The sample was stained with the DAPI (4',6-diamidino-2-phenylindole)/Antifade (1:10,000, Thermo Fisher Scientific) for nuclear staining.

\section{Statistical analysis}

The experimental data (tumor volume, necrosis volume, survival time) were expressed as the mean \pm standard deviation (SD). The student $t$-test was used to compare the difference between two groups and ANOVA (analysis of variance) was used to compare the differences between three groups. The Kaplan-Meier analysis with log-rank test was used to compare the survival between the three groups. A $P$-value less than 0.05 was considered as significantly different.

\section{Results}

\section{The advanced stage glioma model was successfully implanted}

The pathology of the tumors from rats in the three groups was examined by histology staining. The tumors from all three groups demonstrated pathological features that were consistent with grade IV according to the World Health Organization glioma classification 21 days postimplantation (Figure 1). Microscopically, the features of advanced nature of the glioma were seen in histology analysis, such as the foci of necrosis with pseudopalisading of anaplastic cells, mitotic and dense nuclei, and microvascular endothelial cell proliferation.

\section{Cytolysin A gene expression was inducible in the implanted glioma}

Bioluminescence imaging study showed that the engineered S. typhimurium presented in both tumor and reticuloendothelial tissues on the first day of IV injection of bacteria (Figure 2A).
A

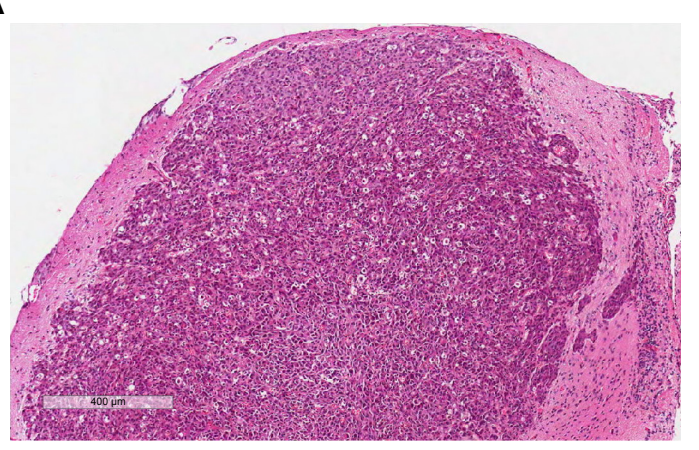

B

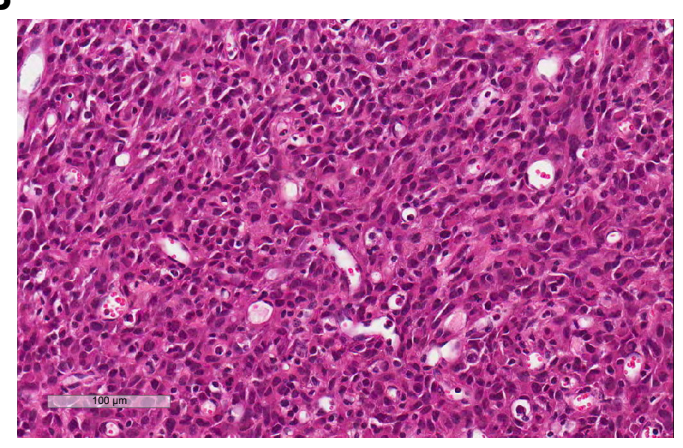

Figure I H\&E histology analysis of the gliomas in advanced stage 21 days postimplantation.

Note: Scale bar: (A) $400 \mu \mathrm{m}$; (B) $100 \mu \mathrm{m}$.

Abbreviation: $\mathrm{H} \& \mathrm{E}$, hematoxylin-eosin. 
A

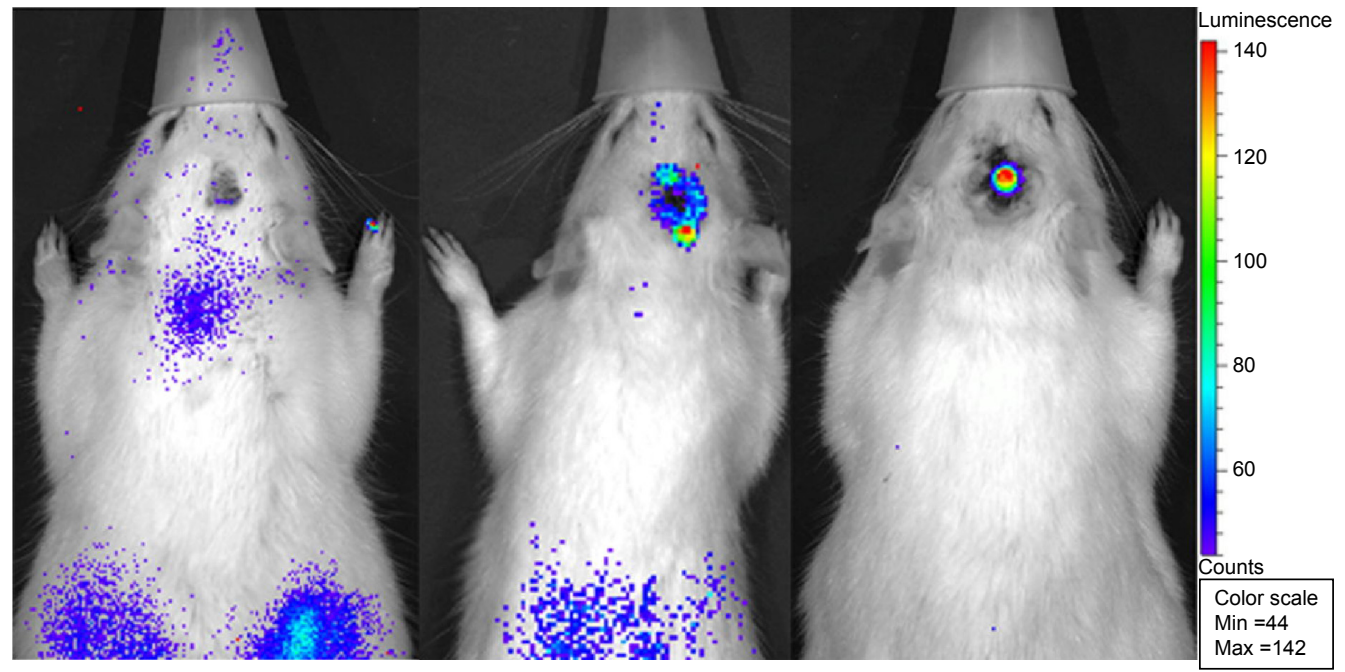

B

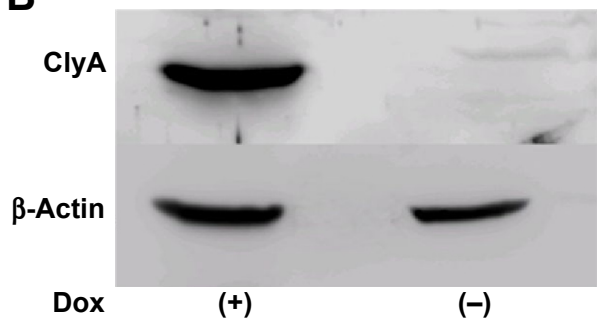

C
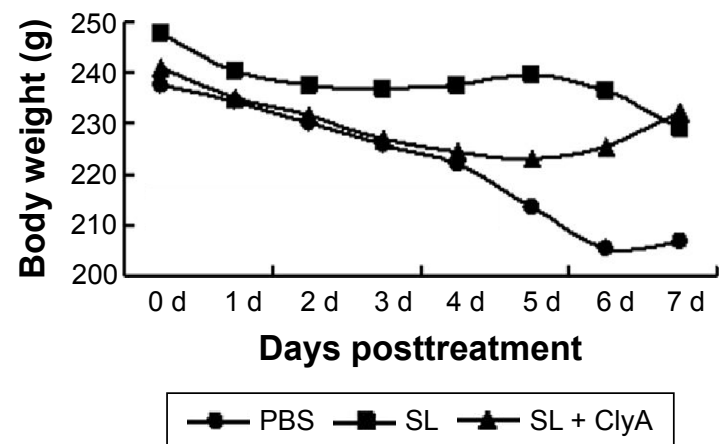

Figure 2 The distribution of the inducible ClyA gene expression.

Notes: (A) Bioluminescence images were obtained following IV injection of the engineered Salmonella typhimurium before doxycycline administration. Expression of luxCDABE in the advanced gliomas was verified by the in vivo bioluminescence imaging at I, 2, and 3 days after IV injection of the engineered S. typhimurium. (B) Immunoblotting analysis of the expression of ClyA in tumor tissues from rats injected with engineered S. typhimurium before (-) and after doxycycline induction (+). (C) The observed body weights of rats were not significantly different between the three groups at the first 7 days of treatment. $P>0.05$.

Abbreviations: ClyA, cytolysin A; IV, intravenous; Dox, doxycycline; PBS, phosphate-buffered saline; SL, treatment group; d, days.

However, the fluorescence signal concentrated in glioma tumor but disappeared in the reticuloendothelial tissues on the third day of IV injection of bacteria (Figure 2A). To confirm that ClyA is inducible upon doxycycline administration, we performed Western blot analysis that successfully detected the presence of ClyA protein in the tumor tissues only after doxycycline administration (Figure 2B). The body weights of rats were not significantly different between the three groups, suggesting that expression of ClyA in tumors by IV administration of engineered S. typhimurium did not elicit severe toxicity (Figure 2C).

\section{Engineered S. typhimurium reduced tumor volume and induced tumor necrosis}

MRI study demonstrated that the glioma tumor volume was significantly increased 21 days postimplantation, suggesting successful implantation of glioma (Figure $3 \mathrm{~A}$ and $\mathrm{B}$ ). There were no significant difference in tumor volume and tumor necrosis volume before adding doxycycline. The average tumor volumes of the SL + ClyA group, SL group, and control group were $186.4 \pm 12.7 \mathrm{~mm}^{3}, 187.8 \pm 15.4 \mathrm{~mm}^{3}, 177.6 \pm 10.8 \mathrm{~mm}^{3}$, respectively at day $21(P>0.05)$. The average necrosis volumes of the SL + ClyA group, SL group, and control group were $26.1 \pm 3.7 \mathrm{~mm}^{3}, 27.7 \pm 5.2 \mathrm{~mm}^{3}, 24.5 \pm 4.3 \mathrm{~mm}^{3}$, respectively, at day $21(P>0.05)$ (Figure 3E). However, the tumor volume of the SL + ClyA group was significantly reduced compared with the other two groups 7 days post-doxycycline treatment or 28 days postimplantation (control: $278.7 \pm 28.1$ $\mathrm{mm}^{3}$; SL: $253.2 \pm 27.1 \mathrm{~mm}^{3}$; SL + ClyA: 201.5 $\pm 20.4 \mathrm{~mm}^{3}$, $P<0.05$, Figure $3 \mathrm{C}$ and $\mathrm{D})$. In addition, the necrotic tumor volume significantly increased in the SL + ClyA group 7 days after doxycycline treatment compared to the other two groups (control: $37.8 \pm 4.9 \mathrm{~mm}^{3}$; SL: $94.7 \pm 9.2 \mathrm{~mm}^{3}$; SL + ClyA: $98.7 \pm 12.6 \mathrm{~mm}^{3}, P<0.01$, Figure $3 \mathrm{~F}$ ). 

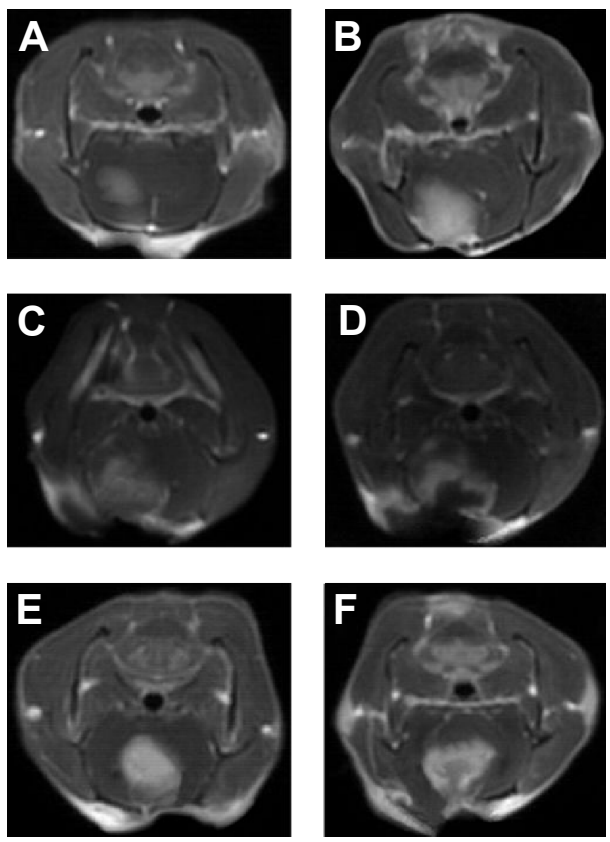

G

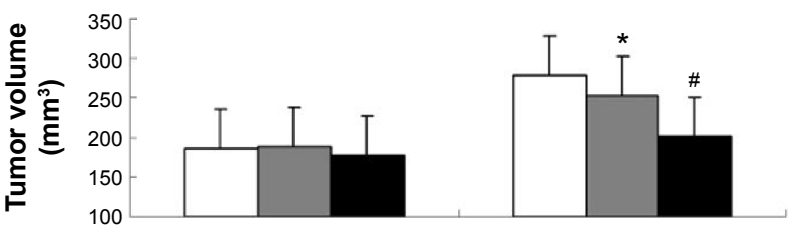

21

Days posttreatment

H

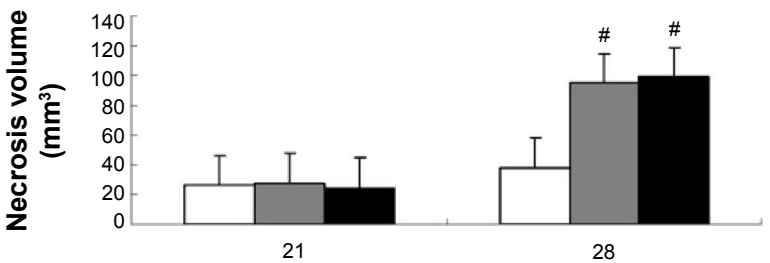

Days posttreatment

PBS

$\mathrm{SL} \mathrm{SL}+\mathrm{ClyA}$

Figure 3 Engineered Salmonella typhimurium significantly reduced glioma tumor volume by inducing necrosis.

Notes: Contrast-enhanced TI weighted image showed the implanted glioma tumors in control and SL group at: (A, C) 2 I days postimplantation, (B, D) 28 days postimplantation, (E) before doxycycline treatment, and (F) after doxycycline treatment. (G) Tumor volumes were compared between the three groups at 21 days postimplantation and 7 days post-doxycycline treatment ( 28 days postimplantation). $(\mathbf{H})$ Necrosis volumes were compared between the three groups at $2 \mathrm{I}$ days postimplantation and 7 days postdoxycycline treatment (28 days postimplantation). $* P<0.05$ versus the control group, ${ }^{\sharp} P<0.01$ versus the control group.

Abbreviations: PBS, phosphate-buffered saline; SL, treatment group; ClyA, cytolysin A.

\section{Engineered S. typhimurium acted on both tumor core and proliferative outer layer in advanced glioma}

Histopathological studies showed that the engineered S. typhimurium induced large area of necrosis in the tumor center 28 days postimplantation (Figure 4A). Interestingly, immunofluorescence staining revealed that the engineered S. typhimurium mainly accumulated in the necrotic zones of glioma. However, the ClyA protein was detected in the outer proliferative layer of the tumor (Figure 4B-E), suggesting that $S$. typhimurium bacteria had its antitumor function in the tumor core while the ClyA protein secreted by the engineered S. typhimurium can penetrate into the proliferative zone to inhibit tumor growth.

\section{Engineered S. typhimurium therapy improved survival in rats with advanced glioma}

The survival benefit of the engineered S. typhimurium therapy in the rat glioma model is shown in Figure 5. The control group has a median survival period of 29 days. The median survival for the SL group and the SL + ClyA group was 32 and 39 days, respectively, which are significantly prolonged compared to the control group $(P<0.01)$.

\section{Discussion}

Since Coley observed that some cancer patients were cured of their tumors following postoperative bacterial infection in the middle of last century, there were more and more preclinical studies on bacterial therapy of cancer. ${ }^{20}$ Recently, studies have reported the obligate anaerobes such as Bifdobacterium and Clostridium in the treatment of cancer in mouse models. ${ }^{21}$ However, the obligate anaerobes can only grow in the necrotic zone of tumors, which limits their antitumor efficacy. ${ }^{21}$ S. typhimurium is a facultative anaerobic bacteria which can overcome the above disadvantage, since it can penetrate into tumor regions that are inaccessible to passive therapies by self-propulsion. ${ }^{13}$ In addition, S. typhimurium has been found to colonize in tumors. ${ }^{22,23}$ In the past decade, numerous studies have demonstrated that the efficacy of S. typhimurium in the treatment of many types of malignancies. ${ }^{8,9,12,22-28}$

After being infected with S. typhimurium, the host immune system was stimulated by bacteria components to cause necrotizing shock and even death. Therefore, $S$. typhimurium often need to be attenuated to be used in cancer treatment. The best known attenuated S. typhimurium strains for bacterial tumor therapy is the msbB-mutant strain with a modified lipid A moiety (VNP20009) and the A1-R strain. ${ }^{8,9,27,28}$ Although the Phase I clinical trial have shown 
A

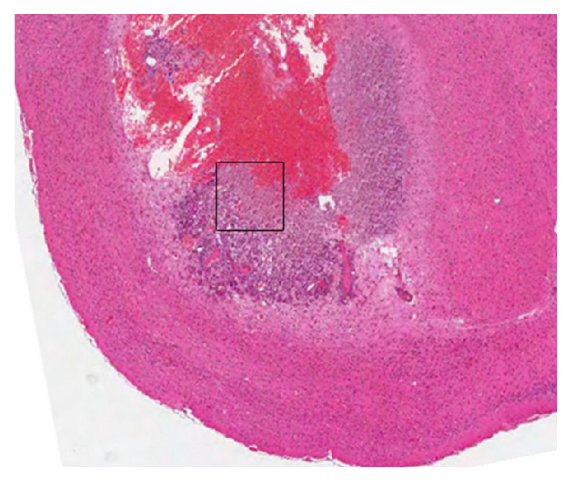

C

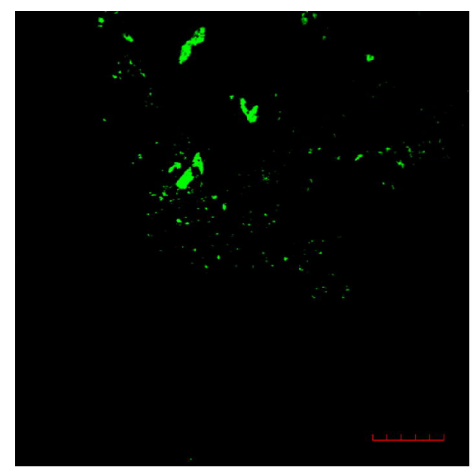

D

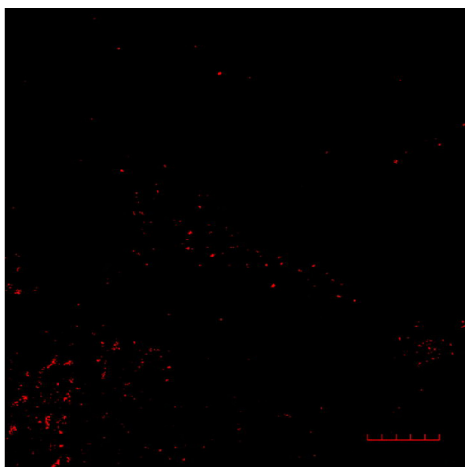

B

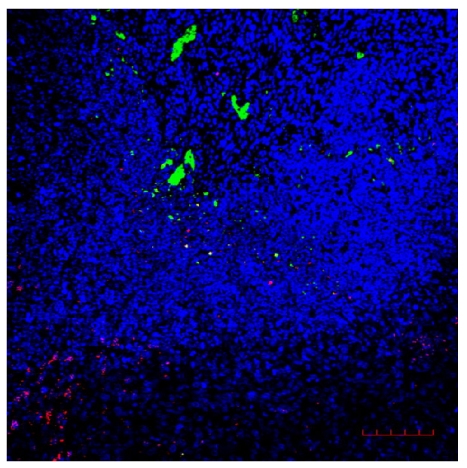

E

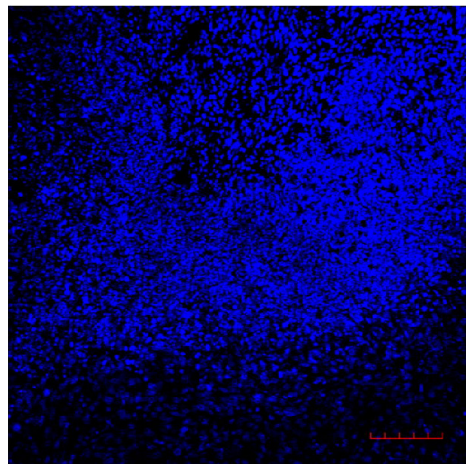

Figure 4 Engineered Salmonella typhimurium has antitumor roles in both tumor core and outer proliferative zone.

Notes: (A) H\&E staining of the glioma infected by the engineered S. typhimurium 28 days postimplantation. The black box included the border area of the necrotic tumor core and the proliferative zone in the SL + ClyA group. (B) A merged image of the immunofluorescent staining of the tissue from the black box (A) with (C) antisalmonella antibody (green), (D) anti-ClyA antibody (red), and (E) DAPI/Antifade (blue). Scale bar $=100 \mu \mathrm{m}$.

Abbreviations: H\&E, hematoxylin-eosin; SL, treatment group; ClyA, cytolysin A; DAPI, 4',6-diamidino-2-phenylindole.

the safety profile of bacterial therapy, the tumor colonization by the bacteria were disappointingly limited, perhaps due to the over-attenuation. ${ }^{29}$ In this study, we used the guanylyl-5'diphosphate-3'-diphosphate-deficient S. typhimurium strain ( $\Delta$ ppGpp S. typhimurium). This strain can reduce the bacterial toxicity by $10^{6}$ times and, meanwhile, can effectively elicit

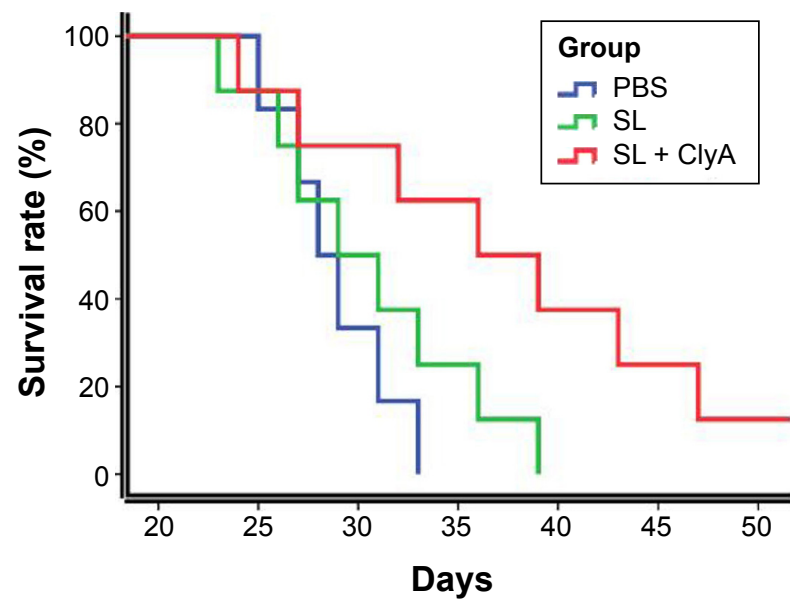

Figure 5 The survival rates of the control, SL, and SL + ClyA groups. Note: $P<0.01$.

Abbreviations: PBS, phosphate-buffered saline; SL, treatment group; ClyA, cytolysin A. both systemic and mucosal antibody responses by silencing relA and spoT genes as well as inducing the cell-mediated immunity response..$^{15,30}$

Because the bacterial gene can be easily manipulated, S. typhimurium can be engineered specifically for cancer therapy. In this study, we selected ClyA from a wide range of cancer-killing proteins to equip the S. typhimurium. ClyA is known as a pore-forming toxin synthesized by Escherichia coli $\mathrm{K}-12$ and other enteric bacteria. ClyA-encoded toxin can promote pore forming on the target cell membrane and induce apoptosis in host cells with extensive destruction effects. ${ }^{31}$ ClyA also has the ability to induce apoptosis in macrophages, which means ClyA is not only cytotoxic to tumor but also cytotoxic to normal tissue, ${ }^{12,15}$ especially in the reticuloendothelial tissues of liver and spleen due to their initial colorization in these organs after tail vein injection. ${ }^{13}$ Therefore, it is crucial to control the production of ClyA only at the appropriate time and location. The doxycyclineinducible expression system is a suitable regulatory system that enables tight control of $C l y A$ gene expression in tumors. Doxycycline is not toxic in mammalian cells and has no inhibitory effects on bacterial growth at the concentration required for gene expression induction. ${ }^{12}$ In addition, 
doxycycline has a long history of safe clinical use in humans. Therefore, doxycycline is a safe and effective induction agent for regulation of gene expression in vivo. ${ }^{11}$

In addition, to know how to control the expression of the cytotoxic cytolysin, we also need to know when to induce its expression. Since bacteria initially localize at the reticuloendothelial organs, ${ }^{13}$ ClyA should only be induced when the bacteria accumulates in the targeted tumor but not in peripheral normal tissues. To achieve this purpose, we employed a bioluminescent enzyme named luxCDABE (lux) which was integrated into the chromosome of S. typhimurium. lux is a perfect imaging gene because its light source is from the luciferin-luciferase reaction, in which no external excitation source is required for luminescence and thus reduces background noise signals. ${ }^{13,32}$ In this study, we found that the bioluminescence of lux gene can be stably expressed in $S$. typhimurium. The relative number of luminescent bacteria can be obtained through the intensity of the signal. In addition, we can monitor the in vivo bacterial distribution in real time. We found that the bacteria have almost cleared from the reticuloendothelial organs and accumulated in the glioma 3 days after IV injection of bacteria. Upon administration of doxycycline, the observed body weights of treated rats were not significantly different, which meant that the induction of ClyA in gliomas in engineered Salmonella with the TetR promoter system and bioluminescent enzyme was safe and tolerable.

In order to evaluate the therapeutic value of the engineered Salmonella in advanced glioma, we employed the contrast-enhanced T1WI-MRI to assess the tumor extent and treatment effects. The enhancing portion of the lesions corresponds to regions of the tumor cell proliferation and could be used to distinguish between the proliferating tumor zone and necrotic tumor zone. ${ }^{33,34}$ In our study, the contrastenhanced T1WI-MRI had shown that the tumor necrosis volume in the SL + ClyA group was significantly higher than that in the control group. The histological analysis showed that $S$. typhimurium coexisted with necrotic tissues but was rarely observed in viable tumor area, indicating that the Salmonella can induce tumor necrosis and apoptosis. The underlying mechanism may be related to the induction of tumor necrosis factor alpha by S. typhimurium. ${ }^{22}$ This unique characteristic of $S$. typhimurium therapy to kill tumor from inside would reduce damage to normal tissues. However, it means that the bacteria are mainly accumulated in the immunity quarantined area $^{35}$ and are absent from the outer layer tumor tissue in which the tumor cells rapidly proliferate and invade surrounding tissues. Consistently, we found that it is difficult to control the rapidly spreading glioma in the advanced stage by $S$. typhimurium alone in the SL group. ${ }^{7,12}$ ClyA protein can penetrate through the barrier comprised of the host neutrophils that are responsible for keeping Salmonella from spreading. Histological analysis in our study indicated that ClyA was found at the proliferating region of the tumor tissue, which means it was expressed by the engineered bacteria and was able to reach the proliferating area of the gliomas. This phenomenon can explain why the bacteria + ClyA group has a better efficacy than the bacteria alone group ${ }^{7}$ in reducing tumor volume.

In addition, it has been reported that the efficacy of engineered bacteria treatment via the tail vein was not good as intracranial injection, mainly due to the poor penetration ability of $S$. typhimurium to the BBB in IV injection. ${ }^{8,9}$ However, this problem did not exist in our study with advanced glioma model. That is because the BBB has been severely damaged in rats with advanced glioma, so bacteria can freely reach the brain tumor and uniformly distribute in the tumor upon IV injection.

In conclusion, we used the engineered bacteria to treat the advanced glioma in rats with promising results. Our study demonstrated that $S$. typhimurium- $\Delta \mathrm{p} \mathrm{G}^{\text {lux/pT-ClyA }}$ effectively inhibited the tumor growth and resulted in necrosis in advanced stage of glioma. The glioma cells were highly sensitive to $S$. typhimurium- $\Delta \mathrm{pG}^{\mathrm{lux} / \mathrm{pT} \text {-ClyA }}$ therapy, which significantly reduced the tumor volume and prolonged survival of the rats. We employed the TetR promoter system to prevent ClyA toxicity to normal tissue. Taken together, the engineered bacteria are a novel and effective treatment strategy for advanced glioma.

\section{Acknowledgments}

We would like to thank Hainan Natural Science Foundation (No 814372) and Key Science and Technology Plan Projects of Hainan (ZDXM20130067) for funding support.

\section{Disclosure}

The authors report no conflicts of interest in this work.

\section{References}

1. Ostrom QT, Gittleman H, Farah P, et al. CBTRUS statistical report: primary brain and central nervous system tumors diagnosed in the United States in 2006-2010. Neuro Oncol. 2013;15 Suppl 2:ii1-ii56.

2. Stupp R, Mason WP, van den Bent MJ, et al. Radiotherapy plus concomitant and adjuvant temozolomide for glioblastoma. NEngl JMed.2005;352(10): 987-996.

3. Stupp R, Hegi ME, Mason WP, et al. Effects of radiotherapy with concomitant and adjuvant temozolomide versus radiotherapy alone on survival in glioblastoma in a randomised phase III study: 5-year analysis of the EORTC-NCIC trial. Lancet Oncol. 2009;10(5):459-466. 
4. Scott CB, Nelson JS, Farnan NC, et al. Central pathology review in clinical trials for patients with malignant glioma. A report of Radiation Therapy Oncology Group 83-02. Cancer. 1995;76(2):307-313.

5. Minchinton AI, Tannock IF. Drug penetration in solid tumours. Nat Rev Cancer. 2006;6(8):583-592.

6. St Jean AT, Zhang M, Forbes NS. Bacterial therapies: completing the cancer treatment toolbox. Curr Opin Biotechnol. 2008;19(5):511-517.

7. Forbes NS. Engineering the perfect (bacterial) cancer therapy. Nat Rev Cancer. 2010;10(11):785-794.

8. Momiyama M, Zhao M, Kimura H, et al. Inhibition and eradication of human glioma with tumor-targeting Salmonella typhimurium in an orthotopic nude-mouse model. Cell Cycle. 2012;11(3):628-632.

9. Kimura H, Zhang L, Zhao M, et al. Targeted therapy of spinal cord glioma with a genetically modified Salmonella typhimurium. Cell Prolif. 2010;43(1):41-48.

10. Wen M, Jung S, Moon KS, Jiang SN, Li SY, Min JJ. Targeting orthotopic glioma in mice with genetically engineered Salmonella typhimurium. J Korean Neurosurg Soc. 2014;55(3):131-135.

11. Williams KJ, Joyce G, Robertson BD. Improved mycobacterial tetracycline inducible vectors. Plasmid. 2010;64(2):69-73.

12. Jiang SN, Park SH, Lee HJ, et al. Engineering of bacteria for the visualization of targeted delivery of a cytolytic anticancer agent. Mol Ther. 2013;21(11):1985-1995.

13. Min JJ, Kim HJ, Park JH, et al. Noninvasive real-time imaging of tumors and metastases using tumor-targeting light-emitting Escherichia coli. Mol Imaging Biol. 2008;10(1):54-61.

14. Flentie KN, Qi M, Gammon ST, et al. Stably integrated luxCDABE for assessment of Salmonella invasion kinetics. Mol Imaging. 2008;7(5): 222-233.

15. Nguyen VH, Kim HS, Ha JM, Hong Y, Choy HE, Min JJ. Genetically engineered Salmonella typhimurium as an imageable therapeutic probe for cancer. Cancer Res. 2010;70(1):18-23.

16. Grommes $\mathrm{C}$, Landreth GE, Sastre M, et al. Inhibition of in vivo glioma growth and invasion by peroxisome proliferator-activated receptor gamma agonist treatment. Mol Pharmacol. 2006;70(5):1524-1533.

17. Song M, Kim HJ, Kim EY, et al. ppGpp-dependent stationary phase induction of genes on Salmonella pathogenicity island 1. J Biol Chem. 2004;279(33):34183-34190.

18. Massoud TF, Gambhir SS. Molecular imaging in living subjects: seeing fundamental biological processes in a new light. Genes Dev. 2003; 17(5):545-580.

19. Chang HR, Comte R, Pechere JC. In vitro and in vivo effects of doxycycline on Toxoplasma gondii. Antimicrob Agents Chemother. 1990;34(5): 775-780.

20. Coley WB. The treatment of malignant tumors by repeated inoculations of erysipelas. With a report of ten original cases. 1893. Clin Orthop Relat Res. 1991;(262):3-11.
21. Kimura NT, Taniguchi S, Aoki K, Baba T. Selective localization and growth of Bifidobacterium bifidum in mouse tumors following intravenous administration. Cancer Res. 1980;40(6):2061-2068.

22. Leschner S, Westphal K, Dietrich N, et al. Tumor invasion of Salmonella enterica serovar Typhimurium is accompanied by strong hemorrhage promoted by TNF-alpha. PLoS One. 2009;4(8):e6692.

23. Kasinskas RW, Forbes NS. Salmonella typhimurium lacking ribose chemoreceptors localize in tumor quiescence and induce apoptosis. Cancer Res. 2007;67(7):3201-3209.

24. Zhao M, Geller J, Ma H, Yang M, Penman S, Hoffman RM. Monotherapy with a tumor-targeting mutant of Salmonella typhimurium cures orthotopic metastatic mouse models of human prostate cancer. Proc Natl Acad Sci U S A. 2007;104(24):10170-10174.

25. Nagakura C, Hayashi K, Zhao M, et al. Efficacy of a geneticallymodified Salmonella typhimurium in an orthotopic human pancreatic cancer in nude mice. Anticancer Res. 2009;29(6):1873-1878.

26. Zhao M, Suetsugu A, Ma H, et al. Efficacy against lung metastasis with a tumor-targeting mutant of Salmonella typhimurium in immunocompetent mice. Cell Cycle. 2012;11(1):187-193.

27. Low KB, Ittensohn M, Le T, et al. Lipid A mutant Salmonella with suppressed virulence and TNFalpha induction retain tumor-targeting in vivo. Nat Biotechnol. 1999;17(1):37-41.

28. Zhao M, Yang M, Li XM, et al. Tumor-targeting bacterial therapy with amino acid auxotrophs of GFP-expressing Salmonella typhimurium. Proc Natl Acad Sci U S A. 2005;102(3):755-760.

29. Toso JF, Gill VJ, Hwu P, et al. Phase I study of the intravenous administration of attenuated Salmonella typhimurium to patients with metastatic melanoma. J Clin Oncol. 2002;20(1):142-152.

30. Na HS, Kim HJ, Lee HC, Hong Y, Rhee JH, Choy HE. Immune response induced by Salmonella typhimurium defective in ppGpp synthesis. Vaccine. 2006;24(12):2027-2034.

31. Ralph ET, Guest JR, Green J. Altering the anaerobic transcription factor FNR confers a hemolytic phenotype on Escherichia coli K12. Proc Natl Acad Sci U S A. 1998;95(18):10449-10452

32. Hoffman RM, Zhao M. Whole-body imaging of bacterial infection and antibiotic response. Nat Protoc. 2006;1(6):2988-2994.

33. Earnest Ft, Kelly PJ, Scheithauer BW, et al. Cerebral astrocytomas: histopathologic correlation of MR and CT contrast enhancement with stereotactic biopsy. Radiology. 1988;166(3):823-827.

34. Tynninen O, Aronen HJ, Ruhala M, et al. MRI enhancement and microvascular density in gliomas. Correlation with tumor cell proliferation. Invest Radiol. 1999;34(6):427-434.

35. Westphal K, Leschner S, Jablonska J, Loessner H, Weiss S. Containment of tumor-colonizing bacteria by host neutrophils. Cancer Res. 2008;68(8) 2952-2960.
OncoTargets and Therapy

\section{Publish your work in this journal}

OncoTargets and Therapy is an international, peer-reviewed, open access journal focusing on the pathological basis of all cancers, potential targets for therapy and treatment protocols employed to improve the management of cancer patients. The journal also focuses on the impact of management programs and new therapeutic agents and protocols on

\section{Dovepress}

patient perspectives such as quality of life, adherence and satisfaction. The manuscript management system is completely online and includes a very quick and fair peer-review system, which is all easy to use. Visit http://www.dovepress.com/testimonials.php to read real quotes from published authors. 